\title{
Microneurosurgical Management of Intractable Temporal Lobe Epilepsy (TLE) from Mesial Temporal Sclerosis (MTS) by Amygdalohippocampectomy plus Standard Anterior Temporal Lobectomy: A Report of Three Cases
}

\author{
FORHAD HOSSAI N CHOWDHURY, ${ }^{1}$ MD. RAZIUL HAQUE, ${ }^{2}$ MD. SHAFIQUL ISLAM, ${ }^{1}$ ASIT CHANDRA SARKER, ${ }^{1}$ AFM \\ MOFAKKHARULISLAM ${ }^{3}$
}

\begin{abstract}
:
Intractable Temporal Lobe Epilepsy may be due to Mesial Temporal Sclerosis (MTS), tumors [i.e. low grade glioma, Arterio-venous Malformation (AVM) etc], trauma, infection (Tuberculosis) etc. Intractable Temporal Lobe Epilepsy (TLE) from Mesial Temporal Sclerosis (MTS) is usually resistant to antiepileptic drugs and surgery is the treatment of choice like other form of TLE. MTS is the most common cause of intractable epilepsy in adult. Here we report three cases of surgically treated TLE that were due to MTS in the department of neurosurgery, Dhaka Medical College Hospital and a private Hospital, Dhaka, Bangladesh from August 2009 to February 2010. In all cases the only presenting symptoms was complex partial seizure (Psychomotor epilepsy) for which all underwent scalp EEG (Electro Encephalogram) and MRI (Magnetic Resonance Imaging) of Brain. All patients were managed by amygdalohippocampectomy plus standard anterior lobectomy. All patients are seizure and disease free till last follow up.
\end{abstract}

Keyword: Intractable temporal lobe epilepsy, Mesial temporal sclerosis, Microneurosurgical management, Amygdalohippocampectomy.

\section{Introduction:}

The definition of drug resistant and intractable epilepsy is not absolutely fixed. About 20\%-30\% epilepsies are drug resistant and intractable. ${ }^{1,2}$ In this group of patient some form of surgery is usually needed either to cure epilepsy or to improve living standard by better control of epilepsy with drugs.

Hippocampus, alveus, fimbria, sabiculum, dentate gyrus and parahippocampal gyrus are jointly called mesial temporal lobe. Amygdala is sometime added in mesial temporal lobe. MTS is usually resulted from birth asphaxia (mild to severe) or hypoxia from any cause. Epilepsy from MTS is usually intractable and disabling which demands surgical treatment. The result of such type of epilepsy surgery usually brings happiness to patient, patient's family, epileptologist and to surgeon. The idea of surgical treatment for epilepsy is not new. However, widespread use and general acceptance of this treatment has only been achieved during the past three decades. ${ }^{3}$ Tremendous improvements in imaging resulted in an increased ability for preoperative identification of intracerebral and potentially epileptogenic foci and lesions. High resolution magnetic resonance imaging plays a major role in structural imaging. EEG (Especially video), MRI and other functional imaging usually help in identification of epileptogenic zone or foci. ${ }^{3}$ Today, epilepsy surgery is more effective and conveys a better seizure control rate from better understanding of pathophysiology of epilepsy along with unbelievable advancement in microneurosurgery. It has become safer and less invasive, with lower morbidity and mortality rates. ${ }^{3}$

We have just started intractable epilepsy surgery in Bangladesh. Here we report our initial experience (of three cases) of intractable epilepsy surgery (amygdalohippocampectomy) from MTS.

\section{Case presentation:}

\section{Case 1:}

A 13 years old girl presented with sudden staring followed by unconsciousness and left sided tonic-clonic convulsion involving left upper and lower limbs since birth. All these

1. Neurosurgeon, Dept. of Neurosurgery, Dhaka Medical college Hospital, Dhaka.

2. Associate Professor, Dept. of Neurosurgery, Dhaka Medical college Hospital, Dhaka.

3. Associate Professor, Dept. of Medicine, SSMC \& Mitford Hospital, Dhaka

Correspondence: Chowdhury Forhad Hossain, Department of Neurosurgery, Dhaka Medical College Hospital. E-mail: forhadchowdhury@yahoo.com 
events persisted for 40-50 seconds .She had history of mild birth asphyxia. Her family history of such kind of illness is negative. Her parents visited several physicians both in the country and abroad. She took several AEDs (Antiepioleptic drugs) in combination for several years without significant improvement in seizure control. There was 25 -30 seizure attack per day even with multiple AEDs (Carbamazipine + Phenobarbitone+ Valproic acid). Scalp EEG revealed abnormal epileptic spikes originating from right centro-temporal region. MRI of brain showed features of right mesial temporal sclerosis (MTS) i.e right temporal horn apparent dilatation, decreased hippocampal volume and punctuate hyperintensity in hippocampus) (Figure1).

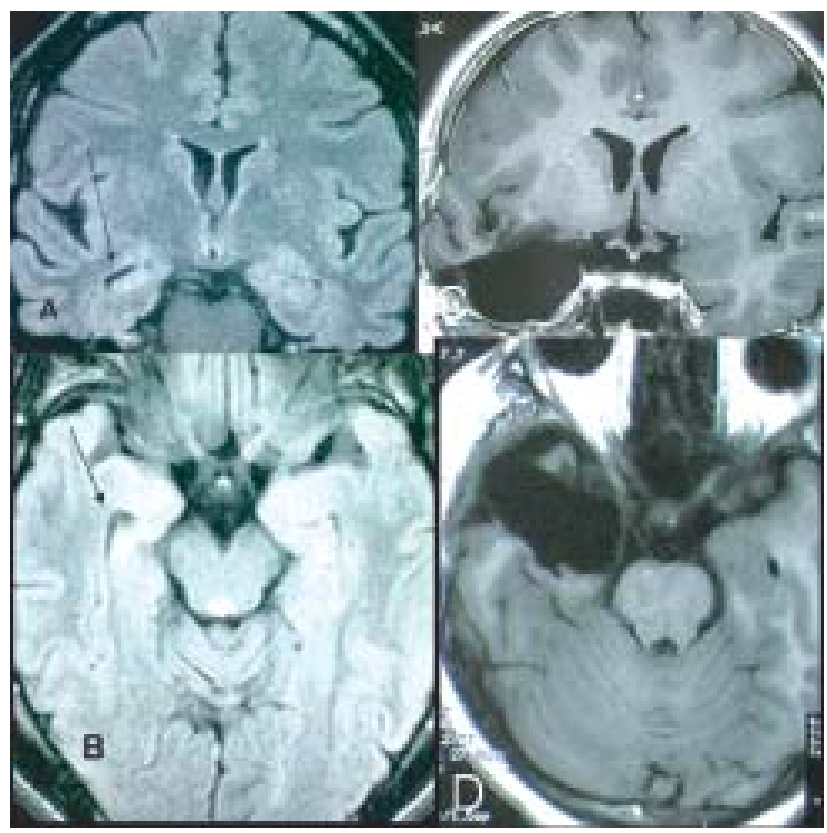

Fig.-1: MRI of brain (FLAIR) coronal image (Fig 1 A) and Axial image (Fig 1 B) showing right mesial temporal sclerosis (MTS) with compensatory dilatation of temporal horn in comparison to left temporal horn. MRI of brain T1 weighted images coronal and axial view respectively after amygdalohippocampectomy and standard anterior temporal lobectomy. (Fig 1 C,D)

She underwent right amygdalohippocampectomy plus standard anterior temporal lobectomy. Postoperatively she recovered uneventfully. She was put on carbamazipine 100 mg three times daily that was tapered over 4 months after operation. She is seizure free for last six month with carbamazipine 100mg 12 hourly.

\section{Case 2:}

A 27 years old right handed female presented with epigastric discomfort and nausea followed by loss of awareness of

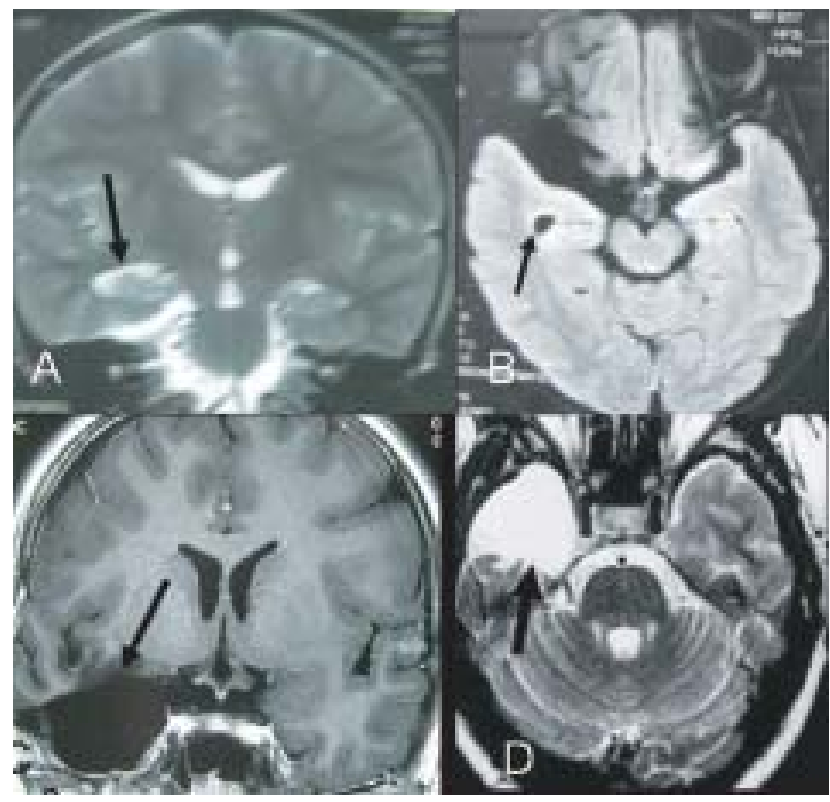

Fig.- 2: MRI of brain (Fig 2 A) Axial mage (FLAIR) \& Coronal image(T2W) (Fig 2B) showing right mesial temporal sclerosis (MTS) with compensatory dilatation of temporal horn in comparison to left temporal horn with anterior temporal arachnoid cyst and right temporal hypoplasia. MRI of brain T2 weighted axial image and $T 1 W$ coronal image respectively after amygdalohippocampectomy with excision of arachnoid cyst and standard anterior temporal lobectomy. (Fig.-2 C,D)

surroundings along with fall and generalized tonic clonic convulsion (without tongue bite, fecal and urinary incontinence) for a period 90-120 seconds for last 21 years. She had history of repeated hospital admission for head injury resulting from fall. Initially this event of attack was less frequent (1-2 attack/week).She visited several of physicians and took many AEDs in different dose with different combination without any improvement in seizure disability. When she visited us, she was on high dose of three AEDs (Carbamazipine + Sodium valproite + Phenoytoin). But frequency of seizure was increasing (15-20 attack/day). She had history of moderate birth asphyxia. She was hyperactive and emotionally labile with saliva dribbling from angle of her mouth, she had multiple scar marks on face, forehead, scalp and shoulder from fall during epileptic attacks. Her intelligence was low but could just maintain her daily personal activities with the help of an assistant. Memory function could not be assessed properly. Her general, systemic and other neurological examination was normal. Scalp EEG showed epileptogenic focus in right temporal region. MRI of brain showed generalized mild cortical atrophy with features of right MTS. She underwent right amygdalohippocampectomy plus standard anterior temporal 
lobectomy. Postoperatively she recovered uneventfully. She was put on carbamazipine $100 \mathrm{mg}$ three times a day. She had one seizure attack two weeks after operation and another attack two month after operation. Then she has been seizure free for last five months. Her hyperactivity is still present but in a reduced form. Post operative MRI of brain at the end of $4^{\text {th }}$ months after operation showed features of removal of mesial temporal lobe with standard anterior temporal lobectomy. Postoperatively assessment for visual field defect or nominal aphasia and memory dysfunction could not be done.

\section{Case-3:}

A 12 years old boy presented with sudden transient global headache, nausea followed by unconsciousness for 1-2 minute followed by typical post ictal state for another 3-5 minutes. He was suffering for last five years. Initially there was 12-15 attacks per year that increased to 3-5 attacks per day even with adequate AEDs treatment (Carbamazipine \& Sodium Valproate).His family history and his birth history was normal. Scalp EEG revealed abnormal epileptic spikes originating from right posterior centro-temporal region along with right anterior temporal slowing. MRI of brain showed features of right mesial temporal sclerosis (Right temporal horn apparent dilatation, decreased hippocampal volume and punctuate hyperintensity in hippocampus) and right temporal lobe hypoplasia along with large anterior temporal arachnoid cyst (3x2x3cm) without mass effect (Figure2).

He underwent right amygdalohippocampectomy plus standard anterior temporal lobectomy with excision of arachnoid cyst. Postoperatively she recovered uneventfully. He was put on the same treatment as the other two patients . He has been seizure free for last three month with carbamazipine. He has no clinical or perimetric visual field defect, dysphasia or memory disturbance.

\section{Discussion:}

Epilepsy surgery started about 125 years ago. William MacEwen (1848-1924) and Victor Horsley (1857-1916) in London were the first to localize and remove epileptogenic lesions, as identified by their symptomatogenic zone, according to the pioneering work of John Hughlings Jackson (1835-1911). ${ }^{3}$ After a long dormant stage, epilepsy surgery developed tremendously in last three decade. ${ }^{3}$ Among the drug resistant intractable epilepsy, temporal lobe epilepsy is the commonest epilepsy (about 80\%) and it responds beautifully to surgery. ${ }^{1,3}$ Like obstructive jaundice (which is called surgical jaundice), temporal lobe epilepsy is called the surgical epilepsy. Common causes of intractable surgical epilepsy are cortical dysplasia (especially in children), MTS, temporal lobe and other lobe tumors such as DNET (Dysembryoplastic Neuroepithelial Tumor), ganglioglioma and other, cortical atrophy, stroke, trauma, vascular lesion(AVM, cavernoma )etc. 1,3,4

In case 1 intractable TLE is due to MTS. This girl has history of birth asphyxia, so we can think this MTS was due to birth asphyxia which is a common cause of MTS. In case 2 patient had cerebral palsy with intractable and disabling temporal lobe epilepsy resulted from birth asphyxia. In such a case decision of surgery is difficult as features of cerebral palsy will persist after surgery; but here positive things in favor of surgery are intractable, severely disabling epilepsy with clean cut epileptogenic pathology that usually responds nicely to surgery. In this case another thing to add that amygdalectomy reduced her hyperactivity that is expecting in such a case. In case-3 intractable epilepsy was due to MTS with temporal lobe hypoplasia rather than arachnoid cyst. Epilepsy associated with MTS usually AEDs resistant and needs surgery. Short term result of surgery in these cases is excellent. In these cases combination of AEDs failed to control disabling epilepsy. In most of the cases surgery is curative if there is no second focus or at least good control of epilepsy can be achieved with AED. ${ }^{3,4}$

We went for amygdalohippocampectomy with or without lesionectomy plus standard anterior temporal lobectomy in all cases but selective amygdalohippocampectomy with lesionectomy could be another surgical option to these lesions. ${ }^{3}$ Size of the lesion, possibility of different pathology, narrow space through trans-sylvian rout and possibility of manipulation and damage of critical vessel and neural structures and most importantly less familiarity of the approach along with less success rate of selective amygdalohippocampectomy in controlling epilepsy helped us to take decision in favor of trans middle temporal gyrus approach amygdalohippocampectomy standard anterior temporal lobectomy instead of selective amygdalohippocampectomy in the hope to cure epilepsy as well as the lesion. It has been suggested that the amount of tissue resected in mesiotemporal operations is crucial for surgical success in mesial TLE. ${ }^{5-9}$ In a randomized, prospective study comparing the trans-sylvian with the transcortical approach, seizure outcome was similar. 10,11 Middle temporal gyrus approach does not usually damage the optic radiation (Meyer loop) unless excessive backward and upward retraction or misdirection of transcortical incision. In two cases we found no visual field defect both clinically $\&$ in perimetry and in one case it could not be assessed.

Generally, complication rates of epilepsy surgery are relatively low and thought to be acceptable, with 
approximately 1 to $2 \%$ permanent morbidity. ${ }^{12}$ The rate of minor complications was $3.6 \%$, and the rate of major complications was $1.26 \%$ in the Zürich series of 478 amygdalohippocampectomies. Persisting hemiparesis occurred in $0.84 \%$ as a result of choroidal infarcts of the internal capsule. ${ }^{13}$ Typical neurological complications after surgery for TLE include temporary dysphasia or hemiparesis as caused by manipulation-induced brain swelling or brain contusion, small vessel infarction, and hemorrhage. There are the classic surgical problems such as infection, thrombosis, etc., in the range of 2 to $4 \%$, which rarely cause permanent damage. The mortality rate is clearly below $1 \%$ in most series. $^{2}$

About 70\% patients become seizure free after temporal lobe epilepsy surgery and another $15-25 \%$ patient will have at least a $90 \%$ reduction in seizure frequency. ${ }^{2}$ Seizure control rate in MTS after surgery is excellent. ${ }^{4,11}$ In certain developmental tumors, e.g., gangliogliomas and dysembryoplastic neuroepithelial tumors, this can be treated with excellent seizure-control rates in most patients. ${ }^{14}$ Also, certain types of low-grade gliomas (e.g., isomorphic subtype of low-grade astrocytoma, pilocytic astrocytoma) can be operated on with excellent results. ${ }^{15-19}$ In a series limited to preoperatively tailored resections for lesional (nonsclerotic) mesial TLE, satisfactory seizure control was obtained in 86\% of patients. ${ }^{20}$ Outcome with lesionectomy and corticectomy was excellent, especially when a tumor was present (95\% satisfactory seizure control). ${ }^{21}$ Although it depends more on patient selection than surgery, it should also be noted that operating on children and adolescents with epilepsy is extraordinarily promising with respect to seizure control and neuropsychological and psychosocial outcomes . 22-27 'Engel grading' is used for assessment of outcome of epilepsy surgery. In point of epilepsy control in our cases final comment can not be made as follow up period is still small but in this small follow up period control of seizure is excellent.

\section{Conclusion:}

Epilepsy surgery today is more effective with better seizure control rates; it is safer and less invasive with lower morbidity and mortality rates. Development of 'epilepsy surgery programe' by a team formed of epileptologist, neuropathologist, neurophysiologist, neuroradiologist and neurosurgeon with the facilities of video EEG, MRI and other imaging facilities is very essential for successful development and upgradation of epilepsy surgery. Finally it should be realized that A "bright" epilepsy neurosurgeon is helpless without an epileptologist who will at first get hold of the patients and participate in the management of all types epilepsy including surgical epilepsy.

\section{Conflict of Interest: None}

\section{References:}

1. Greenberg MS. Seizures. In: Greenberg MS, ed. Hand book of Neurosurgery. $5^{\text {th }}$ edition, New York: Thieme, pp 254$284,2001$.

2. Lowenstein DH. Seizures and Epilepsy. In: Fauci AS, Braunwald E, Kasper DL,et al, eds. Harrison's principles of internal medicine, $17^{\text {th }}$ edition. New York: McGraw-Hill, pp 2494-2512.

3. Schramm J, Clusmann H. Surgery of epilepsy. Neurosurgery 2008; 62 (2):463-481

4. Harvey S, Cross JH, Shinnar S, Matheren BW. The ILAE Pediatric Epilepsy Surgery Survey Taskforce : Defining the spectrum of international practice in pediatric epilepsy surgery patients. Epilepsia 2008; 49(1):146-155

5. Awad IA, Katz A, Hahn JF et al. Extent of resection in temporal lobectomy for epilepsy. Interobserver analysis and correlation with seizure outcome. Epilepsia 1989; 30:756762

6. Bonilha L, Kobayashi E, Mattos JP et al. Value of extent of hippocampal resection in the surgical treatment of temporal lobe epilepsy. Arq Neuropsiquiatr 2004; 62:15-20

7. Nayel MH, Awad IA, Luders H. Extent of mesiobasal resection determines outcome after temporal lobectomy for intractable complex partial seizures Neurosurgery 1991; 29:55-61

8. Renowden SA, Matkovic Z, Adams CB et al. Selective amygdalohippocampectomy for hippocampal sclerosis: Postoperative MR appearance. Am J Neuroradiol 1995; 16:1855-1861

9. Wyler AR, Hermann BP, Somes G: Extent of medial temporal resection on outcome from anterior temporal lobectomy. A randomized prospective study. Neurosurgery 1995; 37:982991

10. Lutz MT, Clusmann H, Elger CE et al. Neuropsychological outcome after selective amygdalohippocampectomy with transsylvian versus transcortical approach: A randomized prospective clinical trial of surgery for temporal lobe epilepsy. Epilepsia 2004; 45:809-816

11. Schaller C, Jung A, Clusmann H, Schramm J, Meyer B. Rate of vasospasm following the transsylvian versus transcortical approach for selective amygdalohippocampectomy. Neurol Res 2004; 26:666-670

12. Behrens E, Schramm J, Zentner J, König R. Surgical and neurological complications in a series of 708 epilepsy surgery procedures. Neurosurgery 1997; 41:1-10

13. Wieser HG: Selective amygdalohippocampectomy has major advantages, in Miller JW, Silbergeld DL, eds. Epilepsy Surgery: Principles and Controversies. New York:Taylor \& Francis, 2006, pp 465-478. 
14. Pilcher WH, Silbergeld DL, Berger MS, Ojemann GA. Intraoperative electrocorticography during tumor resection: Impact on seizure outcome in patients with gangliogliomas. J Neurosurg 1993; 78:891-902

15. Blümcke I, Luyken C, Urbach H et al. An isomorphic subtype of long-term epilepsy-associated astrocytomas associated with benign prognosis. Acta Neuropathol (Berl) 2004; 107:381-388

16. Daumas-Duport C, Scheithauer BW, Chodkiewicz JP et al. Dysembryoplastic neuroepithelial tumor: A surgically curable tumor of young patients with intractable partial seizures : Report of thirty-nine cases. Neurosurgery 1988; 23:545-556

17. Luyken C, Blümcke I, Fimmers R et al. The spectrum of long-term epilepsy-associated tumors: Longterm seizure and tumor outcome and neurosurgical aspects. Epilepsia 2003; 44:822-830

18. Morris HH, Matkovic Z, Estes ML et al. Ganglioglioma and intractable epilepsy: Clinical and neurophysiologic features and predictors of outcome after surgery. Epilepsia 1998; 39:307-313

19. Schramm J, Kral T, Kurthen M, Blümcke I. Surgery to treat focal frontal lobe epilepsy in adults. Neurosurgery 2002;51:644-655
20. Clusmann H, Kral T, Fackeldey E et al. Lesional mesial temporal lobe epilepsy and limited resections: Prognostic factors and outcome. J Neurol Neurosurg Psychiatry 2004; 75:1589-1596

21. Schramm J, Kral T, Grunwald T, Blümcke I. Surgical treatment for neocortical temporal lobe epilepsy: Clinical and surgical aspects and seizure outcome.J Neurosurg 2001 ;94:33-42

22. Blume WT. Temporal lobe epilepsy surgery in childhood: Rationale for greater use. Can J Neurol Sci 1997; 24:95-98

23. Clusmann H, Kral T, Gleissner U et al. Analysis of different types of resection for pediatric patients with temporal lobe epilepsy. Neurosurgery 2004; 54: 847-859

24. Gleissner U, Sassen R, Lendt M et al. Pre- and postoperative verbal memory in pediatric patients with temporal lobe epilepsy. Epilepsy Res 2002; 51:287-296

25. Kral T, Kuczaty S, Blümcke I et al. Postsurgical outcome of children and adolescents with medically refractory frontal lobe epilepsies. Childs Nerv Syst 2001; 17:595-601

26. Mathern GW, Giza CC, Yudovin S et al. Postoperative seizure control and antiepileptic drug use in pediatric epilepsy surgery patients: The UCLA experience, 19861997. Epilepsia 1999; 40:1740-1749

27. Westerveld M, Sass KJ, Chelune GJ et al. Temporal lobectomy in children: Cognitive outcome. J Neurosurg 2000; 92:24-30 